\title{
A Memory-Based Evacuation Navigation Model in Complex High-Rise Buildings
}

\author{
Long Xia $\cdot$ Weiguo Song \\ State Key Laboratory of Fire Science, University of Science and Technology of China, \\ Hefei, China, \\ E-mail: qwfzpk@mail.ustc.edu.cn,wgsong@ustc.edu.cn
}

Received: 14 August 2021 / Last revision received: 14 August 2021 / Accepted: 14 September 2021 DOI: $10.17815 / \mathrm{CD} .2021 .123$

\begin{abstract}
In contemporary society, safety issues are the main focus in the field of pedestrian and evacuation dynamics. As for complex high-rise buildings, the navigation strategies of evacuees still need to be further studied. Previous types of research has contributed to the construction of evacuation navigation model in complex high-rise buildings, where pedestrians are regarded as having an omniscient view in most of these models. In reality, evacuees' perception is always limited, especially when the scenario is complex. In this contribution, pedestrians' perception procedure is considered by computing the visible space so that the occlusion of the visual field can be estimated. In addition, human memory progress is modeled. Not all parts of environmental information would be remembered. Driven by evacuees' memory data, a proposed dynamical shortest path algorithm will be periodically implemented or suddenly triggered by incidents during the simulation. For the pedestrians who have no enough knowledge about the evacuation scenario, a communication system is utilized so that information can be obtained from well-knowledged pedestrian, and an autonomous way-finding system will be executed when useful information cannot be acquired through near evacuees. For the microscopic perspective, human following and avoidance behavior is modeled. Simulations in two different types of scenarios are conducted. The knowledge level of simulated agents is gradually evolved by in-room free exploration. Results in different conditions show that the proposed memory-based model can reproduce pedestrians' observation, turn-back, communication, and searching behavior. The intense conflict caused by the bidirectional crowd is observed and analyzed. In addition, the effects of knowledge level are investigated. The presented model in this contribution can be promising and useful in safety engineering.
\end{abstract}

Keywords Pedestrian and evacuation dynamics · modeling $\cdot$ high-rise building . cognition · memory 


\section{Introduction}

In contemporary society, safety issues are the main focus in the field of pedestrian and evacuation dynamics. As for complex high-rise buildings, the navigation strategies of evacuees still need to be further studied.

Previous types of research has contributed to the construction of evacuation navigation model in complex high-rise buildings. For the underlying model, Helbing et al proposed a microscopic pedestrian acceleration model, where the interactions between agents and the surroundings are regarded as social forces [1,2]. Yu et al proposed a centrifugal force model [3]. Similar to Helbing et al's work, this model utilizes a new force expression to estimate the interactions between two pedestrians. The same form of driven force was also selected by Chraibi et al [4]. To the discrete models, Burstedde el ta proposed a cellular automaton model to simulate pedestrian movement and Song et al built a multi-grid model $[5,6]$. This kind of agent-based model can only be employed in a discrete space. Although the multi-grid model combines the features of lattice gas model and the concept of the social force model, the discretization of simulation space still makes it difficult to reproduce pedestrians' behavior. Therefore, a continuous microscopic pedestrian model is utilized in this work. As a basement of continuous microscopic pedestrian movement model, the social force model is widely employed in numerous situations. It is common to modify or added evolution rules into the basic social force model. For instance, Zanlungo et al introduced a prediction method to explicitly estimate the next collision [7]. Simulation results of this model indicate that introducing the prediction of conflict is useful to improve the performance of social force model. Considering the usability and the robustness for modification, the proposed model of this contribution would be an extension of the social force model.

In most of the scientific products based on the aforementioned operational models, the simulated pedestrians are regarded as having an omniscient view. However, in reality, evacuees' perception is always limited, especially when the scenario is complex. In this case, it is necessary to take perceptive features into consideration when modeling for a huge simulation scene. Hasegawa et al conducted experiments for the drivers who were using a personal mobility vehicle and built a microscopic model based on the experiment results [8]. In their work, the perception of the drivers and the avoidance behavior was considered. Xue et al built a social force based model to simulate evacuation in view and hearing limited condition [9]. This work reveals the importance of memory mechanism during evacuation. However, the model assumption is too simple to be suitable for a complex scenario. Speak of the effects from memory mechanism, Xia et al proposed a flow field model with memory effect to simulate crowd flow within a room [10]. Their work supports the idea that modeling work is supposed to be conducted under the guidance of human memories. In this contribution, pedestrians' perception procedure is considered by computing the visible space so that the occlusion of the visual field can be estimated. In addition, human memory progress is modeled. Not all parts of environmental information would be remembered. Driven by evacuees' memory data, a proposed dynamical shortest path algorithm will be periodically implemented or suddenly triggered by incidents during the simulation. For the pedestrians who have no enough knowledge about 
the evacuation scenario, a communication system is utilized so that information can be obtained from well-knowledged pedestrian, and an autonomous way-finding system will be executed when useful information cannot be acquired through near evacuees.

For the navigation aspect, Kneidl et al declared that it is reasonable and useful to use sparse navigation graphs for microscopic pedestrian simulation [11,12]. Their findings were supported by experimental results. Kielar et al proposed a pedestrian routing model for graph-based way finding, which is built on cognitive principles [13,14]. Therefore, a graph-based navigation strategy would be reasonable to be utilized in this work, while the Dijkstra shortest path algorithm is implemented to figure out possible evacuation routes.

The remainder of this contribution is organized as follows. In Sec. 2, the details of the proposed model will be introduced. Assumptions and the driven formulas will be explained. Sec. 3 will represent and analyze the simulation results in two types of scenarios. Then we conclude this work in Sec. 4.

\section{Modeling}

The proposed model is composed of four main modules: kinematics model, navigation model, affair model, and cognition model. The overall model framework is given in Fig. 1 where four parts of the model works in coordinate with each other.

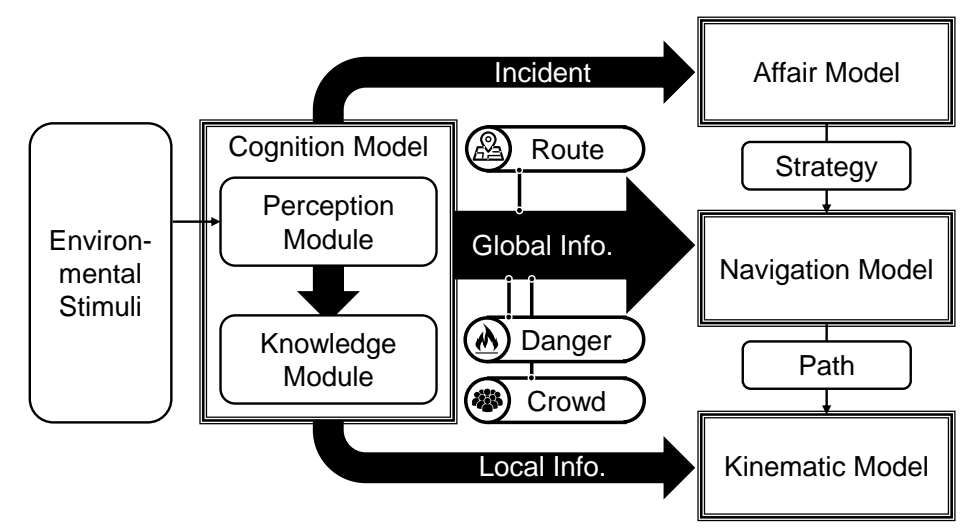

Figure 1 Model framework

The cognition model captures information from the environment and transfers them into agents' memories, as presented in Sec. 2.4. Performed during the whole simulation progress, the cognition model provides basic information which is required by other modules. The major function of the affair model is strategy determination. Affairs containing transaction, touring and evacuation are predefined. Simulated agents would execute different affairs assigned by the affair model and follow the corresponding procedure. Sec. 2.3 contains detailed introduction about the affair model. Introduced in Sec. 2.2, the navigation model plays a vital important role in the evacuation simulation of a highrise building. Rely on the knowledge provided by the cognition model, the navigation model can give agents guidance about the global evacuation route. The kinematics model 
contributes at the base of the deduction model, which drives simulated agents moving towards the target locations and handles the local motion conflicts. The kinematics model will be explained in Sec. 2.1 in details. To obtain a higher computing efficiency, parallel update scheme is utilized where the kinematics and dynamics properties of simulated agents would only be updated when the calculation for the whole loop is accomplished.

\subsection{Kinematics model}

For the kinematics model, the social force model proposed by Helbing et al [1] is modified to be more suitable for the simulation in a complex building. The movement of agents who are simulated using the original social force model is driven by an acceleration equation

$$
\ddot{\boldsymbol{x}}_{i}\left(t_{0}\right)=\frac{v_{i}^{0}(t) \boldsymbol{e}_{i}^{0}(t)-\dot{\boldsymbol{x}}(t)}{\tau_{i}}+\frac{\sum_{j(\neq i)} \boldsymbol{f}_{i j}}{m_{i}}+\frac{\sum_{w} \boldsymbol{f}_{i w}}{m_{i}},
$$

where $\boldsymbol{x}(t)$ denotes the simulated agent $P_{i}$ 's local position at time $t, v_{i}^{0}(t)$ and $\boldsymbol{e}_{i}^{0}(t)$ represents $P_{i}$ 's desired speed and direction respectively, $f_{i j}$ are the interactive forces between $P_{i}$ and any other pedestrian $P_{j}$, and $f_{i w}$ indicate the repulsion from obstacles $O_{w}$ exerted on $P_{i}$.

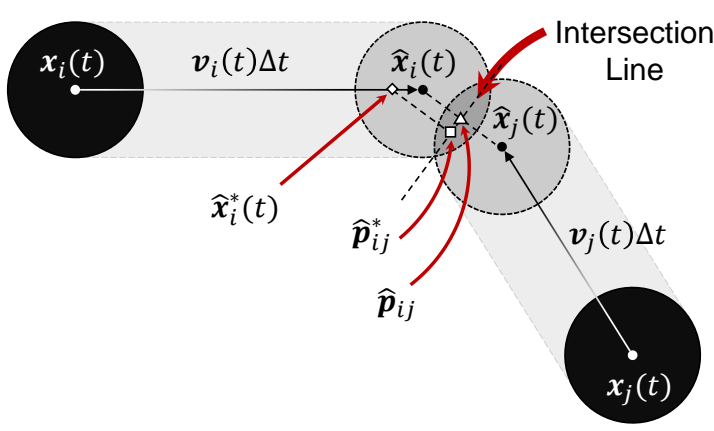

(a)

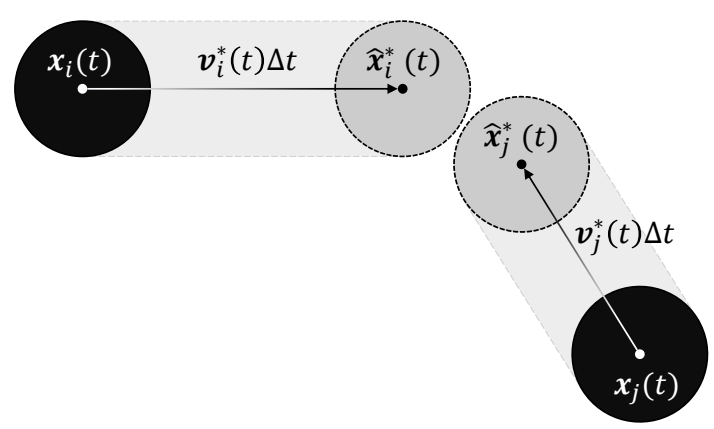

(b)

Figure 2 Conflict handling. (a) Predicted agents' locations and space violation; (b) Modified estimation after conflict handling.

In this contribution, the major framework of the social force model is preserved. Considering that the assumption of strong contact force in social force model is not stable, especially when the simulation scenario is complex, only the non-contact parts are included in the estimation of repulsive forces. Given that a parallel update scheme is utilized in this work, the predicted space violation between agents should be handled after computing the resultant force, so that agents can maximally reach their desired locations without extreme physical contact. In this case, minimal distance assurance is implemented as shown in Fig. 2. In Fig. 2, $\boldsymbol{x}_{i}(t)$ and $\boldsymbol{v}_{i}(t)$ denote pedestrian $P_{i}$ 's location and velocity at time $t, \hat{x}_{i}(t)$ represents the predicted location that satisfies

$$
\hat{\boldsymbol{x}}_{i}(t)=\boldsymbol{x}_{i}(t)+\boldsymbol{v}_{i}(t) \Delta t,
$$


and $\hat{\boldsymbol{p}}_{i j}$ is the intersection point of two predicted agents' intersection line and connecting line. To avoid physical contact between $P_{i}$ and $P_{j}, P_{i}$ should be re-located to $\hat{\boldsymbol{x}}_{i}^{*}(t)$ so that the intersection point $\hat{\boldsymbol{p}}_{i j}$ would be relocated along the intersection line to $\hat{\boldsymbol{p}}_{i j}^{*}$ satisfying

$$
\left\{\begin{array}{l}
\left(\hat{\boldsymbol{x}}_{i}(t)-\hat{\boldsymbol{p}}_{i j}\right) / /\left(\hat{\boldsymbol{x}}_{i}^{*}(t)-\hat{\boldsymbol{p}}_{i j}^{*}\right) \\
\left|\hat{\boldsymbol{x}}_{i}^{*}(t)-\hat{\boldsymbol{p}}_{i j}^{*}\right| \geq r_{i}
\end{array} .\right.
$$

Then $v_{i}(t)$ can be re-computed by

$$
\boldsymbol{v}_{i}^{*}(t)=\frac{\hat{x}_{i}^{*}(t)-\boldsymbol{x}_{i}^{*}(t)}{\Delta t} .
$$

Considering that non-contact forces in the social force model are designed to mimic pedestrians' spontaneous dispersion behavior, extra social forces are defined to reproduce pedestrians' following and avoiding behavior. Eq. 1 will be modified to the following formula

$$
\ddot{\boldsymbol{x}}_{i}\left(t_{0}\right)=\frac{v_{i}^{0}(t) \boldsymbol{e}_{i}^{0}(t)-\dot{\boldsymbol{x}}(t)}{\tau_{i}}+\frac{\sum_{j(\neq i)} \boldsymbol{f}_{i j}}{m_{i}}+\frac{\sum_{w} \boldsymbol{f}_{i w}}{m_{i}}+\frac{\sum_{j \in \boldsymbol{J}_{i}} \boldsymbol{f}_{i j}^{a t t r}}{m_{i}},
$$

where $J_{i}$ includes all the other headway agents who are in the perceptive area of $P_{i}$ as shown in Fig. 3, and $\boldsymbol{f}_{i j}^{a t t r}$ represents the attractive force caused by $P_{j}$ who is moving in similar direction as $P_{i}$ 's. Note that $\boldsymbol{f}_{i j}^{a t t r}$ would become repulsive force if $P_{i}$ and $P_{j}$ are moving in different directions.

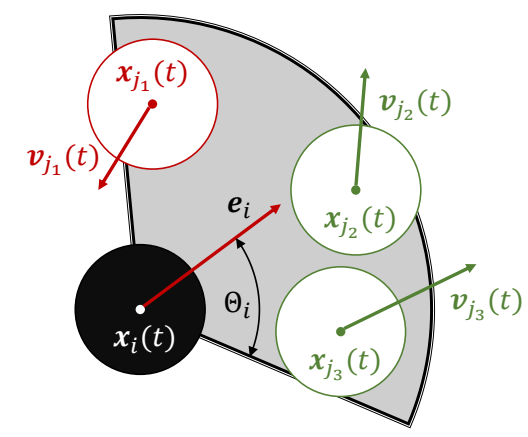

Figure 3 Perceptive area of $P_{i}$. Considering the angular difference between $\boldsymbol{e}_{i}$ and $\boldsymbol{v}_{j}(t), P_{j}\left(j \in \boldsymbol{J}_{i}=\right.$ $\left.\left\{j_{1}, j_{2}, j_{3}\right\}\right)$ presented as green disks would exert attractive force on $P_{i}$ while the red ones would push $P_{i}$ away.

Under aforementioned assumptions, the specific form of each term in Eq. 5 is given as follows.

$$
\boldsymbol{f}_{i j}=A_{i} \exp \left[\left(r_{i j}-d_{i j}\right) / B_{i}\right] \boldsymbol{n}_{i j},
$$

where $A_{i}=2 \times 10^{3} N$ and $B_{i}=0.08 m$ are suggested by Helbing et al [1]. $\boldsymbol{n}_{i j}$ is the normalized vector pointing from $P_{j}$ to $P_{i}$. The formula of non-contact force from obstacle $O_{w}$ 
is

$$
\boldsymbol{f}_{i w}=A_{i} \exp \left[\left(r_{i j}-d_{i w}\right) / B_{i}\right] \boldsymbol{n}_{i w} .
$$

Similarly, $\boldsymbol{n}_{i w}$ here is the normalized vector pointing from $O_{w}$ to $P_{i}$. For the extra attractive force, we have

$$
\begin{gathered}
f_{i j}^{a t t r}=\alpha_{i j}\left\{A_{i}^{a t t r} \exp \left[\left(r_{i j}-d_{i w}\right) / B_{i}\right] \boldsymbol{n}_{i j}\right\}, \\
\alpha_{i j}=\exp \left[-k\left(\boldsymbol{e}_{i} \cdot \boldsymbol{e}_{j}+1\right)\right]-\exp \left[k\left(\boldsymbol{e}_{i} \cdot \boldsymbol{e}_{j}-1\right)\right],
\end{gathered}
$$

where $A_{i}^{\text {attr }}=500 \mathrm{~N}$ with which suitable distance among agents can still be reproduced while reasonable following and avoiding behavior can be observed. $k=10$ is chosen to ensure that the amplitude of $f_{i j}^{a t t r}$ will attenuate more than $60 \%$ when the angular difference between $\boldsymbol{e}_{i}$ and $\boldsymbol{e}_{j}$ is larger than $60^{\circ}$.

\subsection{Navigation model}

Before the shortest path algorithm can be deployed to figure out suitable navigation paths for simulated agents, the indoor map of simulation scenario should be discretized into a graph $G=(V, E)$. In consideration of geometry and functionality, the simulation scenario with three rooms and a flight of stairs can be discretized into eight small rooms, which are presented as polygons in Fig. 4(a) with black solid lines and green dashed lines as the boundary. Fig. 4(b) shows the connectivity of the given layout, where the circular markers demonstrate sub-rooms and the diamond-shaped markers represent passable doors. Since there can be more than one exit in a specific room, the navigation in a single room should also be handled. Therefore, doors in the layout will all be regarded as the vertices of the connected graph built in this contribution, as shown in Fig. 4(c).

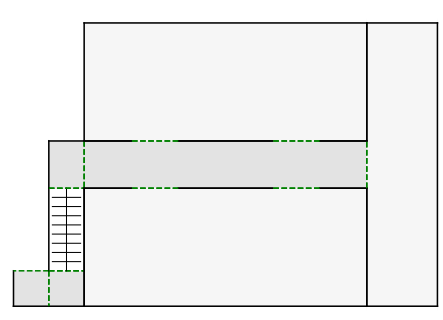

(a)

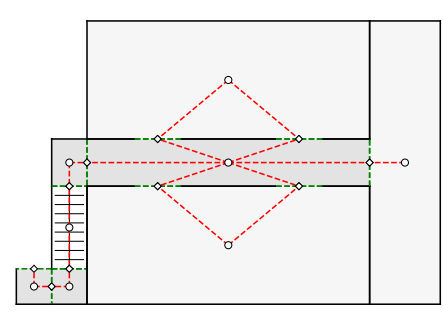

(b)

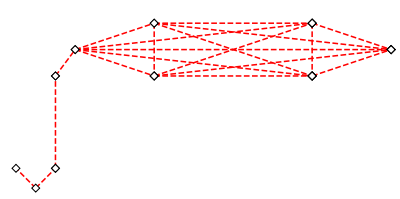

(c)

Figure 4 Discretization of a simulation scenario in two-dimensional view. (a) is the layout of a simulation scenario. (b) shows the discretization and the connectivity of the layout. (c) presents the connected graph.

For a more realistic situation, the simulation scenario would be three-dimensional so that agents can move through floors. As presented in Fig. 5, a multi-floor building can also be discretized into sub-rooms. The connection between different floors is supported 
by stairs. In Fig. 5(b), the cubic markers demonstrate sub-rooms and the diamond-shaped markers represent passable doors.

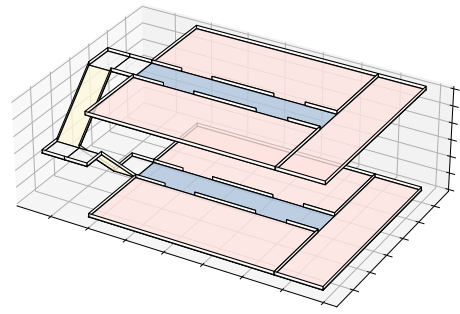

(a)

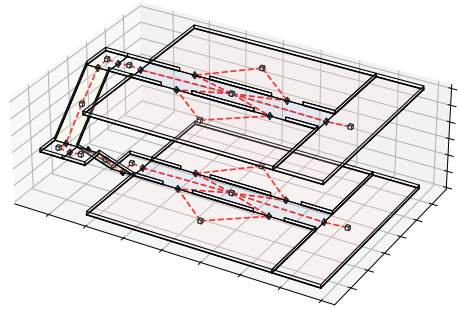

(b)

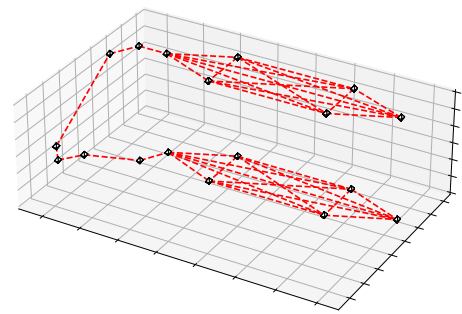

(c)

Figure 5 Discretization of a simulation scenario in three-dimensional view. (a) is the layout of a simulation scenario. (b) shows the discretization and the connectivity of the layout. (c) presents the connected graph.

Whether in two-dimensional scenes or in three-dimensional scenes, the discretization method can transform a complex navigation problem into a heuristic graph search problem. After the aforementioned discretization process, the indoor map of a complex building can be transformed into a undirected graph, where doors of the building are regarded as vertices of the graph. Note that, each agent will maintain its own graph. Let $G_{i}=\left(V_{i}, E_{i}\right)$ be $P_{i}$ 's undirected graph obtained by the discretization method, where $V_{i}=V_{i}^{P} \cup V_{i}^{D} . V_{i}^{P}=\left\{v_{i}^{P}\right\}$ and $V_{i}^{D}$ represent the vertices of the agent and doors, respectively. Here, $V_{i}^{P}$ and the corresponding edges should be dynamically added to $G_{i}$ before the navigation process, which indicating the location of $P_{i}$ itself. The tuple representing an edge can be described as $e_{i, m n}=\left(v_{i, m}, v_{i, n}\right)$, where $e_{i, m n} \in E_{i}, v_{i, m} \in V_{i}$, and $v_{i, n} \in V_{i}$.

In this work, an undirected graph based Dijkstra shortest path algorithm is utilized to navigate simulated agents. To improve the applicability of the Dijkstra shortest path algorithm, the edges of the connected graph are weighted based on both the geometry features and the social features:

$$
w\left(e_{i, m n}\right)=\beta_{1}\left\|\boldsymbol{x}\left(v_{i, m}\right)-\boldsymbol{x}\left(v_{i, n}\right)\right\|_{2}+\beta_{2}\left[N\left(v_{i, m}\right)+N\left(v_{i, n m}\right)\right],
$$

where $\boldsymbol{x}\left(v_{k}\right)$ represents the location of vertex $v_{k}$, and $N\left(v_{k}\right)$ represents the number of agents near to vertex $v_{k}$.

\subsection{Affair model}

As the top level of the proposed memory-based model, the affair model plays a role as policy provider. An affair changing can be triggered whenever $P_{i}$ is exposed to any incident. Several types of affairs and the corresponding trigger conditions are defined as follows.

Routing Routing affair could be triggered whenever there is an incident occurs. For instance, when an accident takes place, so that the agents in the building has to be evacuated. Then $P_{i}$ would route to find a possible evacuation path. Whenever $P_{i}$ enters a new 
sub-room, the routing affair should be triggered, which would be more realistic. If nothing happens during the simulation, the routing affair would also be triggered periodically since people will not blindly follow the previous course. This setup would make simulated agents more agile. The affair would stay silent if $P_{i}$ entered a safe spot, which could decrease the computation cost.

Visiting This affair would be triggered when $P_{i}$ cannot seek a possible route by the navigation model based on its own memories. $P_{i}$ would search in the adjacent room so that new information about the layout could be added to its memories. Therefore, $P_{i}$ could find out more passable routes and connected rooms. This will help $P_{i}$ solve a solution to the exit of the building.

Communication Communication will only occur between agent $P_{i}$ and agent $P_{j}$ who are near to each other. It could be triggered periodically or be triggered when $P_{i}$ is in a visiting status. Because when $P_{i}$ lost its way out, it would be urgent to get information from others so that it might be possible for $P_{i}$ to figure out new path to the exit. Note that, not all the communication would be successful. Therefore, there is a possibility that $P_{i}$ may be unable to get any information from $P_{j}$ after the communication.

\subsection{Cognition model}

The cognition model in this contribution is composited with two parts: perception and knowledge. For the perceptive part of this work, sight and hearing are selected to be the main approaches to gather sensory information of the environment.

As shown in Fig. 6, the simulated agent $P_{i}$, that is displayed as a black disk, would only interact with the ones ( $P_{j}^{a c t i v e}$, presented as red disks) who are near to $P_{i}$ due to the theory of proxemics. Social interactions in this work, like computing the social forces and sharing possible evacuation routes, will not be taken into account between $P_{i}$ and any agent ( $P_{j}^{\text {irrelevant }}$, presented as white disks) who is far away from $P_{i}$. In addition, $P_{i}$ 's vision would be hindered by obstacles like walls (presented as dark lines) and pillars (presented as polygons). If any agent ( $P_{j}^{\text {invisible }}$, presented as a blue disk) who is inside $P_{i}$ 's interesting range (presented as dashed circle with a radius of two meters) but sheltered by obstacles, the interaction between $P_{i}$ and $P_{j}^{\text {invisible }}$ would be ignored.

For the knowledge part, memory is the key feature handled in this contribution. Considering that the layout of the building is discretized into sub-rooms and abstracted into undirected graph (as discussed in Sec. 2.2), each agent would store their own graph to be the support of other models. In addition, they also maintain a time stamp for every node and edge. When two agents communicate with each other, there is a possibility that the newest information about their undirected graph would be updated. It should be noted that the information about agent number, which would be used by the navigation model, is also based on agents' personal perception and memory. Therefore, the assessment about the congestion level of each node is actually unstable, which could be more realistic. 


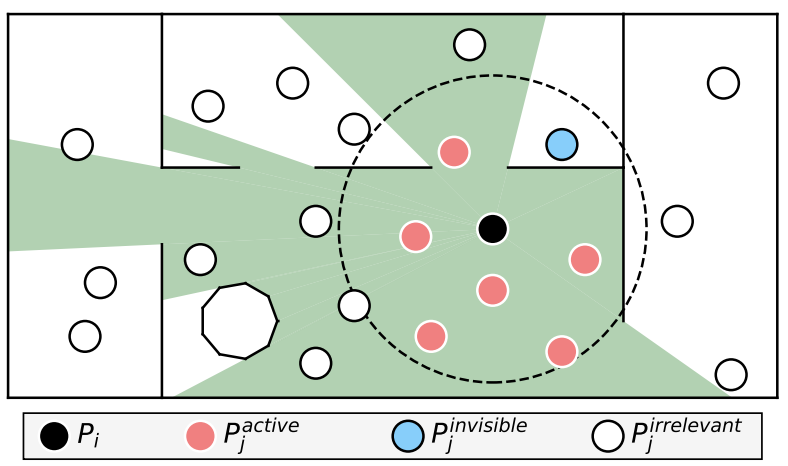

Figure 6 A sketch for $P_{i}$ 's local perception. The green shadow region represents the visible region of $P_{i}$, while the dashed circle shows the range within which $P_{i}$ would be significantly impacted by $P_{j}$.

\section{Simulation results}

A three-story building is designed to be the simulation layout. As shown in Fig. 7(a), an enormous sub-room is used to be the shelter of simulation scene. In this case, when the evacuation starts, simulated agents would try to move to the shelter through the route generated by the dynamic navigation method. In this section, two types of scenarios are defined to validate the applicability of the proposed model and to study the effects from modeling assumptions to simulation results. To obtain a more flexible way finding progress, we set $\beta_{1}=1 \mathrm{~m}^{-1}$ and $\beta_{2}=0.5$, which indicates that if the distance difference between two possible routes is too slight, then the difference of agents' number would greatly change the routing result.

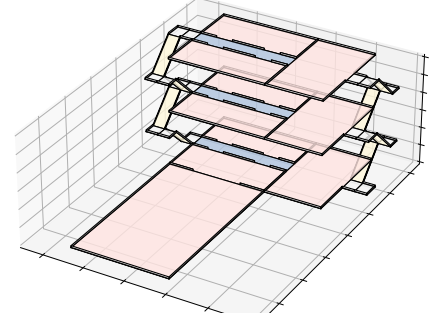

(a)

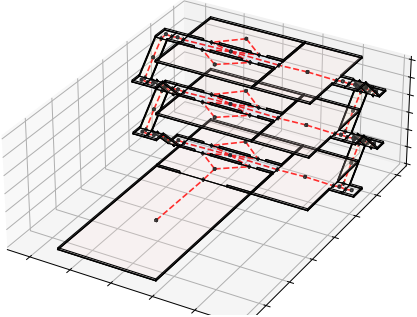

(b)

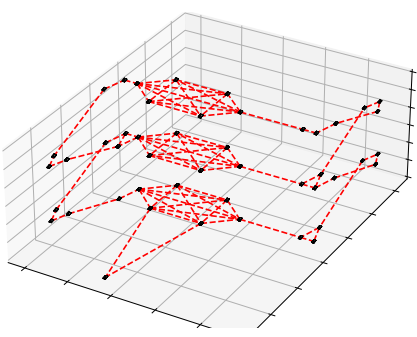

(c)

Figure 7 Simulation layout in three-dimensional view. (a) is the layout of a simulation scenario. (b) shows the discretization and the connectivity of the layout. (c) presents the connected graph.

\subsection{Scenario 1: Blockage in stairwell}

Assuming that some incident occurs in the building blocking up one of the stairwell, which is marked as light red polygon in Fig. 8(a). Therefore, the connection between the first floor and the second floor is broken on the right side of the building. However, the simulated agents who are provided a complete map of the building are not informed about 
the blockage. At the beginning of the simulation in this scenario, all the agents conduct their way finding process based on the complete map where no blockage is recorded. Therefore, a huge number of agents would try to go through the right-side stairwell at the very beginning.

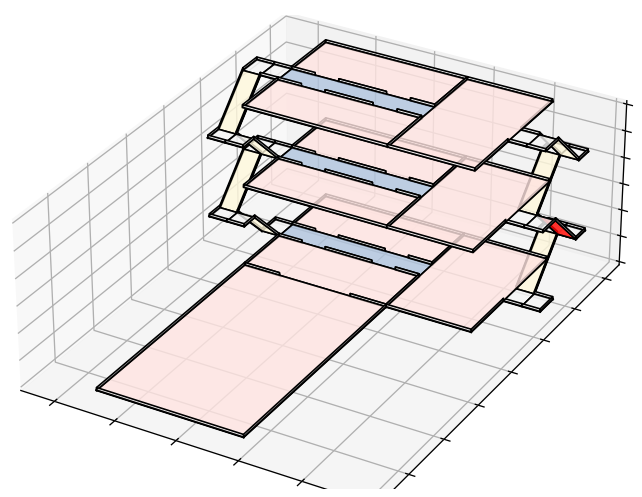

(a)

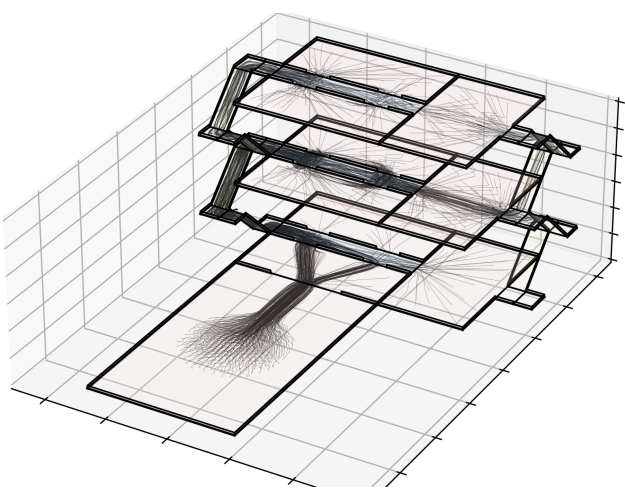

(b)

Figure 8 Layout and simulation trajectories of scenario 1. (a) shows the layout with a blockage. (b) presents the simulation trajectories.

In three-dimensional view, Fig. 8(b) gives the simulation trajectories of more than 300 agents who were randomly settled in the building before the simulation started. It can be observed that a part of agents in the upper right room on the third floor tried to evacuate from the right door when the evacuation started. Then they re-route to choose the left exit because of the navigation consideration on the number of the agents who are near to the door. Similar phenomena can be observed in other places as well. For instance, in the middle hall on the second floor, agents tried to avoid the crowd by entering the adjacent rooms with a longer path. In addition, a part of agents chose to evacuate from the second door of the same room, through which the total evacuation distance would increase. This phenomenon can be observed at the first floor. It is noteworthy that the navigation process triggered by the observation of the stairwell blockage. Agents who were walking in front firstly identified the blockage. However, it is effortful for them to turn around even if the rerouting process has already been handled due to the crowd flow behind, which caused chaos and movement conflict in the stairwell. But this situation did not last long. Benefiting from the communication among agents, the situation of blockage was fast transmitted to the following agents and the crowd flow soon return to stability.

In order to quantify the qualitative description about evacuation conflict among agents, two forms of conflict measurement, $C M_{m}^{t a r}$ and $C M_{m}^{v e l}$ are proposed and utilized. The measurement of velocity conflict, $C M_{m}^{v e l}$, takes agents' instantaneous velocities into account, indicating the difference of movement directions in sub-room $m$. The formula is given as

$$
C M_{m}^{v e l}(t)=1-\frac{1}{N_{\boldsymbol{D}}} \sum_{i, j \in \boldsymbol{D}_{i, m}(t)} \frac{\boldsymbol{v}_{i}(t) \cdot \boldsymbol{v}_{j}(t)}{\left|\boldsymbol{v}_{i}(t)\right|\left|\boldsymbol{v}_{j}(t)\right|},
$$

where set $\boldsymbol{D}_{i, m}(t)$ includes all the agents who are in the room $m$ at time $t$ and $N_{\boldsymbol{D}}$ is the size 
of set $\boldsymbol{D}_{i, m}(t) . \boldsymbol{v}_{i}(t)$ and $\boldsymbol{v}_{j}(t)$ are the velocities of agents $P_{i}$ and $P_{j}$. The other measurement method for routing conflict, $C M_{m}^{t a r}$, describes the difference of agents' temporary moving targets. The computing function is

$$
C M_{m}^{t a r}(t)=\sqrt{\frac{\sum_{i \in \boldsymbol{D}_{i, m}(t)}\left(\Phi_{i, m}(t)-\bar{\Phi}_{m}\right)^{2}}{N_{\boldsymbol{D}}}},
$$

where $\Phi_{i, m}(t)$ demonstrates the indices of $P_{i}$ 's temporary moving targets and $\bar{\Phi}_{m}$ is the average value. Note that $\Phi_{i, m}(t)$ for the same room are re-numbered to be a continuous sequence. For instance, if $P_{0}$, who was firstly detected in room $m$ at time $t$, chose $\boldsymbol{x}_{0}(t)$ as its temporary target, then $\Phi_{0, m}(t)=0$. If $P_{1}$ chose a different target, then $\Phi_{1, m}(t)=1$. If $P_{2}$ chose the same point as $P_{0}$ 's choice, then $\Phi_{2, m}(t)=0$. If $P_{2}$ 's choice is different from $P_{0}$ 's and $P_{1}$ 's, then $\Phi_{2, m}(t)=2$.

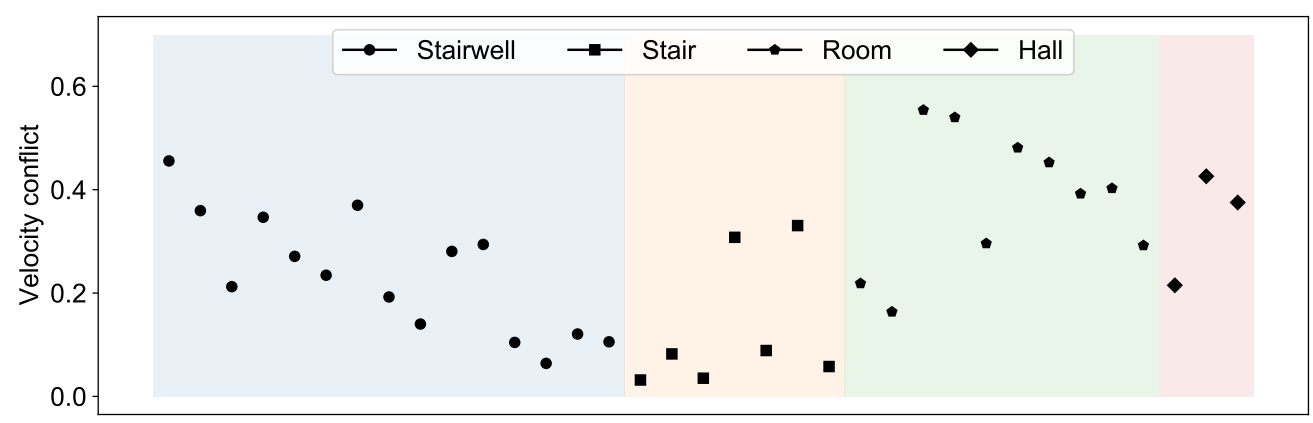

(a)

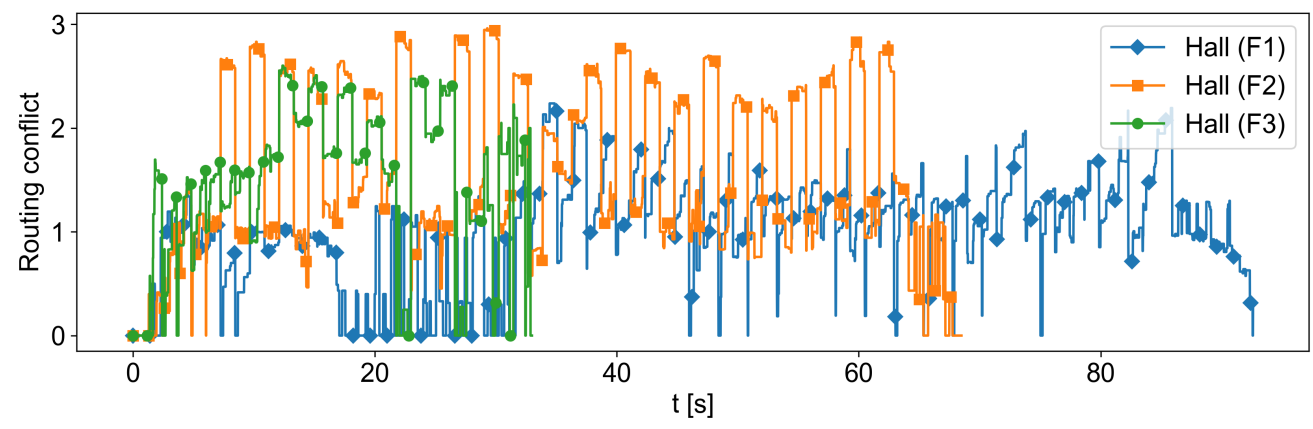

(b)

Figure 9 Conflict measurement. (a) shows the average velocity conflict in different types of building components. (b) shows the routing conflict in the halls on three different floors.

Fig. 9 shows the computing results of two types of conflict measurement. For the velocity conflict, the higher $\left\langle C M_{m}^{v e l}(t)\right\rangle_{t}$ is, the heavier the conflict would be. $\left\langle C M_{m}^{v e l}(t)\right\rangle_{t}$ of stairwells, where there is bidirectional flow, are relatively higher. As shown in Fig. 9(a), the last four scatter points shown in the first part of Fig. 9(a) is lower since these four subrooms belong to the right side of the third floor, where the congestion level is low and rerouting behavior occurs less frequently. It can be observed from the figure, $\left\langle C M_{m}^{v e l}(t)\right\rangle_{t}$ of most of the stairs maintain the lowest value compared with other types of building com- 
ponents. The reason would be that most of the agents will not change their movement directions during the progress of downstairs. However, $\left\langle C M_{m}^{v e l}(t)\right\rangle_{t}$ of two of them are higher than that of the other stairs. One of these two stairs is connected to blockage stairwell. Therefore, a strong conflict would be induced by the confluence flow. The other stair is the connection between the third floor and the second floor, where the congestion level is always high during the whole simulation. $\left\langle C M_{m}^{v e l}(t)\right\rangle_{t}$ for rooms are not only affected by the movement anisotropy but also by the exit selection behavior. However, $<C M_{m}^{v e l}(t)>_{t}$ in the rooms, where there were only a few agents at the beginning of the simulation (for instance, the first two scatter points of the third part in Fig. 9(a)), are still dramatically smaller than those with many agents. Considering that most of the agents entering the first-floor hall were from the above floors and their popular choices would be turning right to enter the room connected to the target room, $\left\langle C M_{m}^{v e l}(t)\right\rangle_{t}$ of the firstfloor hall is relatively lower than that of the other two halls in the second and the third floor (the first scatter point of the fourth part in Fig. 9(a)). For the time evolution of the routing conflict shown in Fig. 9(b), the fluctuations of $C M_{m}^{\text {tar }}(t)$ for the hall at the second floor is dramatically stronger than that of the other two, and the amplitude is also higher, indicating that the re-routing process was performed more frequently there. It could be explained considering that more agents from different floors gathered in this hall during the evacuation simulation. Agents would try to search a faster evacuation path, which is driven by Eq. 10 .

\subsection{Scenario 2: Different knowledge level}

In Sec. 3.1, all the agents were provided the complete map of the layout. As mentioned in Sec. 2.4, agents might only have limited knowledge about the simulation scenario. Under this consideration, we set different ratio for agents' knowledge level to study the effects of perception and communication. For agents who have low level of knowledge, a random ratio from $20 \%$ to $50 \%$ of the information about the layout would be forgotten at the beginning of the simulation. For agents who have high level of knowledge, this ratio would range from $1 \%$ to $20 \%$.

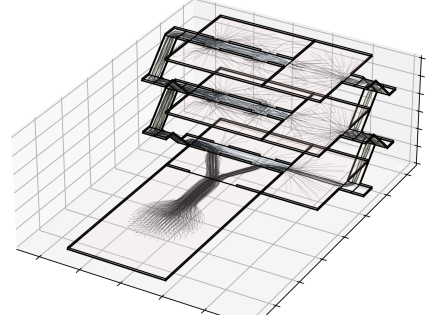

(a)

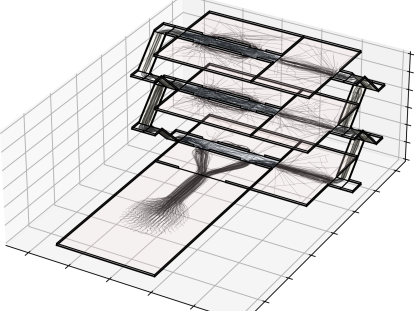

(b)

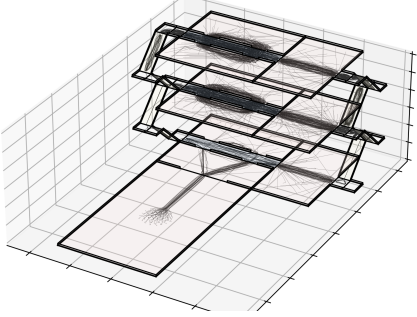

(c)

Figure 10 Simulation trajectories of scenario 2 at 100 seconds. (a) shows the result where all the agents know the complete map at the beginning. (b)shows the result where $0 \%$ of the agents had low knowledge level at the beginning . (c)shows the result where $100 \%$ of the agents had low knowledge level at the beginning. 
Fig. 10 shows the simulation trajectories for scenario 2. Since not all the agents can reach the exit of the building, the trajectories were cut off before 100 seconds. As can be notified from the trajectories, The higher the ratio of low-level-knowledge agents is, the more formless the trajectories would be. The agents with low level of knowledge would search the adjacent room more frequently to try to obtain more information about the layout. In addition, there is no agent has the full map of the simulation layout, although a plenty of agents can still complete the map by sharing information with others. Therefore, they can finally reach the exit of the building, even if the key nodes of the evacuation map were forgotten at the beginning.

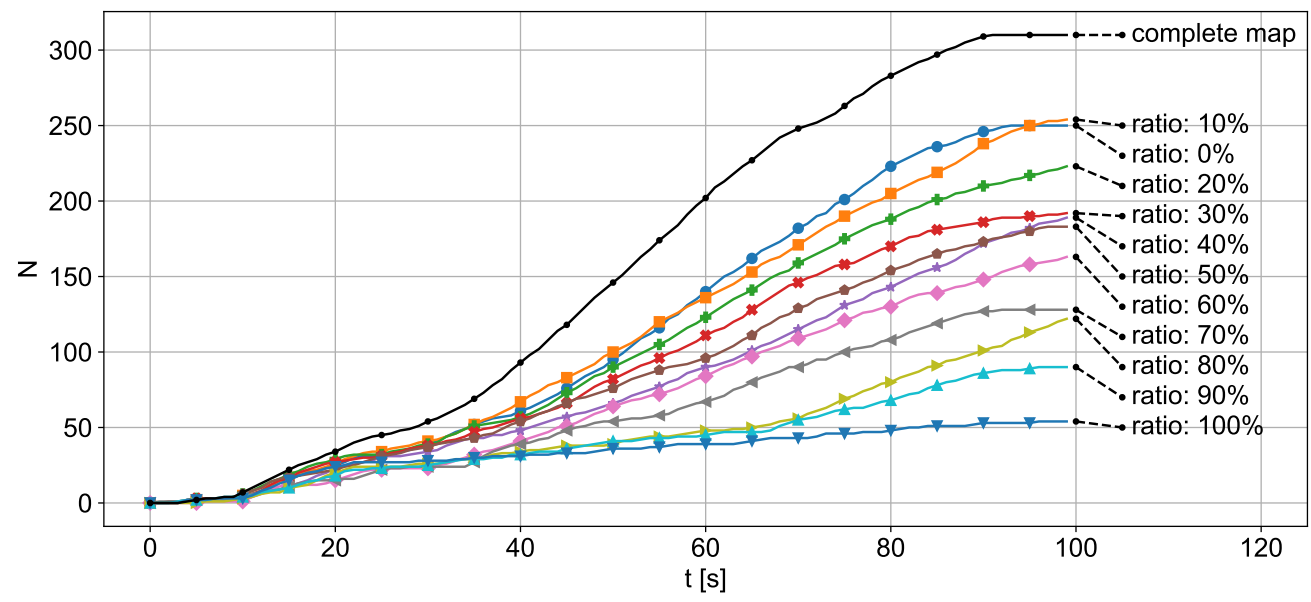

Figure $11 \quad \mathrm{~N}$-t diagram

To further study the effects from the ratio of low-level-knowledge agents, a fundamental diagram is presented in Fig. 11, which describes the relationship between the number of agents who have already escaped from the building and time. As show in Fig. 11, there are four major stages of the $\mathrm{N}$-t curve. In the first stage, agents were about to evacuate, the curve is gentle. Then, the agents who were near to the exit of the building evacuated, resulting an increase of the slope. After that, agents from both sides of the building reached the exit, which makes the curve rise sharply. In the final stage, most of the agents who can evacuate efficiently have already reached the exit. Therefore, the curves of $\mathrm{N}$ t diagram became flatter. Meanwhile, the appearance of the fourth stage indicates that the evacuation capacity of the rest agents stays in a low level so that the safety of the agents are under huge threaten. Comparing the results under different ratio of the lowlevel-knowledge agents, we can find out that the evacuation efficiency will be greatly decreased if the ratio increases. When the ratio equals 50\%, approximately a half of the agents cannot escape from the building in a short time, and when the ratio equals $100 \%$, only one-sixth agents can quickly evacuate before 100 seconds. It should be noted that, in some simulation runs, more agents can still evacuate after an exploration of the building. Some other agents who have already figured out a safe path could be temporarily stuck by the lost crowd, so that they may have the chance to evacuate in the future. In addition, considering that the pruning process of personal map is conducted randomly, while the initialization of simulated agents is also random, the simulation results could 
be slightly different in repetitive group. For instance, in Fig. 11, the N-t curve of ratio $10 \%$ shows more evacuation potential than that of ratio $0 \%$ in this run of simulation. This phenomenon may disappear in other runs.

\section{Conclusion}

In this contribution, a memory-based evacuation navigation model for the evacuation simulation of complex high-rise buildings is proposed, which includes four major submodels: kinematics model, navigation model, affair model and cognition model.

The kinematics model is conducted based on the classical social force model so that the following and avoidance behavior could be considered. In addition, contact forces in original social force model are removed to make the model more stable.

The navigation model can discretize and abstract the simulation layout into undirected graph so that it would be possible to compute a numerical solution for shorted evacuation route based on the Dijkstra algorithm. Distance from agent $P_{i}$ to the doors, distance from one door to another, and the number of agents near the door are chosen to be the weighting metrics of graph edges.

The affair model provides policy to drive the simulated agents. Affairs including routing, visiting and communication are implemented.

The cognition model contains two parts: perceptive module and memory module. Agent $P_{i}$ will autonomously survey the surroundings to gather information about traversability and connectivity. In addition, $P_{i}$ could hear $P_{j}$ 's experience resulting an increase of $P_{i}$ 's knowledge level, so that it could find a better evacuation route. For the memory module, personal undirected graph and graph timestamp are designed to be a numerical representation of human memories.

The proposed model is utilized in two different types of scenarios. In scenario 1, a blockage occurs in one of the stairwell. Agents were not informed about the situation when the evacuation startsed. Although they have the complete map of the building, the blockage should still be found by themselves through perception. Two forms of conflict measurement, $C M_{m}^{t a r}$ and $C M_{m}^{v e l}$, are defined to reveal the conflicts on both kinematic and decision perspectives. Result shows that the process of observing the blockage in the evacuation route would result in strong movement conflicts near the blockage spot, while the communication mechanism can help reduce the conflict and guide the followed agents to avoid the blockage. In addition, re-routing behavior is reproduced. The consideration of the numbers of agent near a door can help the agents avoid unnecessary queuing. In scenario 2, the knowledge level of agents are randomly set. With the increase of the ratio of low-level-knowledge agents, the evacuation efficiency will dramatically decrease. The evacuation efficiency and potential is discussed using N-t fundamental diagram, which numerically reveals the effects from the ratio of low-level-knowledge agents. These simulation results indicate that a consideration of human perception, communication and memory is necessary for a complete evacuation model. Without these setups, evacuation time of the simulations could be short, but the results cannot be realistic. It can also be concluded, the proposed model in this contribution could be promising and useful in safety engineering. 
Acknowledgements This contribution is supported by National Natural Science Foundation of China (52074252), Anhui Key Research and Development Program (202004a07020052).

\section{References}

[1] Helbing, D., Farkas, I., Vicsek, T.: Simulating dynamical features of escape panic. Nature 407(6803), 487-490 (2000). doi : 10 . $1038 / 35035023$

[2] Helbing, D., Molnar, P.: Social force model for pedestrian dynamics. Physical review E 51(5), 4282 (1995). doi: 10.1103 /PhysRevE. 51.4282

[3] Yu, W.J., Chen, R., Dong, L.Y., Dai, S.Q.: Centrifugal force model for pedestrian dynamics. Physical Review E 72(2 Pt 2), 026112 (2005). doi:10.1103/PhysRevE.72.026112

[4] Chraibi, M., Seyfried, A., Schadschneider, A.: Generalized centrifugal-force model for pedestrian dynamics. Physical Review E 82(4), 046111 (2010). doi:10.1103/PhysRevE.82.046111

[5] Burstedde, C., Klauck, K., Schadschneider, A., Zittartz, J.: Simulation of pedestrian dynamics using a two-dimensional cellular automaton. Physica A: Statal Mechanics and its Applications 295(3-4), 507-525 (2001). doi:10.1016/S0378-4371(01)00141-8

[6] Song, W., Xuan, X., Wang, B.H., Ni, S.: Simulation of evacuation processes using a multi-grid model for pedestrian dynamics. Physica A Statistical Mechanics \& Its Applications 363(2), 492-500 (2006). doi:10.1016/j.physa.2005.08.036

[7] Zanlungo, F., Ikeda, T., Kanda, T.: Social force model with explicit collision prediction. EPL (Europhysics Letters) 93(6), 68005 (2011). doi:10.1209/0295-5075/93/68005

[8] Hasegawa, Y., Dias, C., Iryo-Asano, M., Nishiuchi, H.: Modeling pedestrians' subjective danger perception toward personal mobility vehicles. Transportation research part F: traffic psychology and behaviour 56, 256-267 (2018). doi:10.1016/j.trf.2018.04.016

[9] Xue, S., Jia, B., Jiang, R., Shan, J.: Pedestrian evacuation in view and hearing limited condition: The impact of communication and memory. Physics Letters A 380(38), 3029-3035 (2016). doi:10.1016/j.physleta.2016.07.030

[10] Xia, Y., Wong, S., Shu, C.W.: Dynamic continuum pedestrian flow model with memory effect. Physical Review E 79(6), 066113 (2009). 
[11] Kneidl, A., Borrmann, A.: How do pedestrians find their way? results of an experimental study with students compared to simulation results. Emergency Evacuation of people from Buildings (2011)

[12] Kneidl, A., Borrmann, A., Hartmann, D.: Generation and use of sparse navigation graphs for microscopic pedestrian simulation models. Advanced Engineering Informatics 26(4), 669-680 (2012). doi:10.1016/j.aei.2012.03.006

[13] Kielar, P.M., Biedermann, D.H., Kneidl, A., Borrmann, A.: A unified pedestrian routing model for graph-based wayfinding built on cognitive principles. Transportmetrica A: transport science 14(5-6), 406-432 (2018). doi:10.1080/23249935.2017.1309472

[14] Kielar, P.M., Borrmann, A.: Spice: a cognitive agent framework for computational crowd simulations in complex environments. Autonomous Agents and Multi-Agent Systems 32(3), 387-416 (2018). doi: 10.1007 / s1 $0458-018-9383-2$ 